\title{
Evaluation the performance of serum neutrophil gelatinase associated lipocalin as a biomarker of allograft dysfunction in kidney recipients from living donors
}

\author{
Elham Adlravan $^{1}{ }^{\circledR}$, Farid Javandoust Gharehbagh $^{1}{ }^{\circledR}$, Ali Taghizadeh Afshari $^{2}$, Behzad Baradaran ${ }^{3}$, \\ Jaffar Nourooz-Zadeh ${ }^{2,1^{*}}$ \\ ${ }^{1}$ Department of Clinical Biochemistry, Urmia University of Medical Sciences, Urmia, Iran \\ ${ }^{2}$ Nephrology and Kidney Transplant Research Center, Clinical Research Institute, Urmia University of Medical Sciences, Urmia, Iran \\ ${ }^{3}$ Immunology Research Center, Faculty of Medicine, Tabriz University of Medical Sciences, Tabriz, Iran
}

\begin{tabular}{l} 
A R T I C L E I N F O \\
\hline Article Type: \\
Original \\
\hline
\end{tabular}

\section{Article History:}

Received: 22 January 2021

Accepted: 1 August 2021

Published online: 29 August 2021

\section{Keywords:}

Immediate graft function,

Delayed graft function,

Kidney transplantation,

Neutrophil gelatinase associated

lipocalin, Glomerular filtration rate, Creatinine

\begin{abstract}
A B S T RA C T
Introduction: Serum neutrophil gelatinase-associated lipocalin (sNGAL) has been proposed as an early biomarker for the prediction of delayed graft function (DGF) from cadaveric donors.

Objectives: The purposes of this investigation were to explore the time-based trend for sNGAL in kidney recipients from living donors and to evaluate its correlation with graft function recovery during a one-year follow-up.

Patients and Methods: Kidney recipients $(n=39)$ were consecutively enrolled. Sample collection was performed before transplantation and at 2, 16, 24, 36, 48 hours after surgery. Kidney recipients were split into immediate graft function (IGF) and DGF based on estimated glomerular filtration rate (eGFR) on day 5 post-surgery. eGFRs $>60 \mathrm{~mL} / \mathrm{min} / 1.73 \mathrm{~m}^{2}$ on day 5 post-transplantation were considered as IGF. sNGAL was assessed by ELISA. Serum creatinine $(\mathrm{sCr})$ was measured by the Jaffe method.

Results: Rates of participants with IGF or DGF were 25 and 14, respectively. Pre-surgery, sNGAL levels in the DGF subset were $21 \%$ higher than that of the IGF group. At 2-hours checkpoint, area under curve, sensitivity, specificity and cut-off $(\mathrm{ng} / \mathrm{mL})$ for sNGAL were $0.73,100 \%, 52 \%$ and 151.8. sNGAL levels correlated with allograft function at 6,9 and 12 months post-transplantation ( $\mathrm{r}=0.66 ; P=0.007 ; \mathrm{r}=0.836 ; P=0.031$ and $\mathrm{r}=0.93 ; P=0.016$ ).

Conclusion: We have uncovered that monitoring sNGAL in kidney recipients is a useful biomarker for the evaluation of short- and long postoperative outcome in renal transplant patients from living donors. However, multicenter study with large samples-size is required to ascertain the usefulness of sNGAL as diagnostic tool for the evaluation of allograft dysfunction in renal transplant patients from living donors.
\end{abstract}

\section{Implication for health policy/practice/research/medical education:}

Evidence signifies that serum neutrophil gelatinase-associated lipocalin (sNGAL) is a reliable predictor of early graft function organ recipients from deceased donors. We aimed to evaluate the diagnostic performance of sNGAL for the prediction of earlyand long-term graft restoration in kidney recipients from living donors. Organ recipients $(n=39)$ were consecutively enrolled. Serial measurements were performed before transplantation and at 2, 16, 24, 36, 48 hours after surgery. Immediate graft function (IGF) was defined as an estimated glomerular filtration rate (eGFR) $>60 \mathrm{~mL} / \mathrm{min}$ per $1.73 \mathrm{~m}^{2}$ on day 5 post-transplantation. We have uncovered that the value of sNGAL at 2- hours post-surgery correlates allograft function at 6,9 and 12 months posttransplantation. Multicenter study with large samples size is required to ascertain the usefulness of sNGAL as a diagnostic tool for the prediction of allograft function in renal transplant patients from living donors.

Please cite this paper as: Adlravan E, Javandoust Gharehbagh F, Taghizadeh Afshari A, Baradaran B, Nourooz-Zadeh J. Evaluation the performance of serum neutrophil gelatinase associated lipocalin as a biomarker of allograft dysfunction in kidney recipients from living donors. J Renal Inj Prev. 2021; 10(4): e30. doi: 10.34172/jrip.2021.30. 


\section{Introduction}

Kidney transplantation is the most gainful therapeutic option for patients with end-stage renal disease (ESRD) (1). However, the incidence of early delayed graft function (DGF) is still much inferior than desired. Occurrence of DGF varies from $5 \%$ to $50 \%$ depending on organ source (living donors versus cadaveric donors) (2,3). Ischemiareperfusion injuries, immunological responses and donor-related organ quality are the major recognized causes of DGF (4-6). Current clinical indicators of DGF are serum creatinine ( $\mathrm{sCr}$ ) and urine output (UO). The major inherited problem with the $\mathrm{sCr}$ measurement is that the alteration emerges several days after a tubular insult. UO test is a simple and non-invasive index of successful reperfusion but lacks the required functional specificity.

Thus, efforts have been directed towards the search for innovative biomarkers that will facilitate site-specific DGF detection. Pioneering studied have shown that measurement of urine or blood biomarkers such as neutrophil gelatinase associated lipocalin (NGAL), cystatin C, interleukin 18 (IL-18) and kidney injury molecule 1 outperform $\mathrm{sCr}$ in spotting renal dysfunction $(7,8)$. In kidney recipients from cadaveric donors with more than one co-morbid disorder, the marked elevation of sera- and urine NGAL precedes that of sCr $(9,10)$. Additionally, there are correlations between serum neutrophil gelatinase associated lipocalin (sNGAL) and biomarkers of ischemia-reperfusion related kidney injury $(9,10)$. A multicenter investigation involving 225 kidney recipients from brain death donors (The CONTEXT study) revealed that plasma NGAL (pNGAL) predicted early graft dysfunction at day one post-surgery (11). Buemi et al (12) explored the diagnostic performance of pNGAL from kidney recipients involving deceased donors $(\mathrm{n}=80)$ and living donors $(\mathrm{n}=17)$. In the organ recipients from the deceased donors, pNGAL at 24- and 48-hours post-surgery were predictor of initial recovery allograft function. With respect to the organ recipient from the living donors, no conclusion was reached since all kidney recipients were non-DGF.

\section{Objectives}

In this study, we aimed to: 1) revisit the temporal trend of sNGAL of kidney recipients from living donors withand without postoperative complication and 2) evaluate its diagnostic performance to predict allograft recovery at 3-, 6-, 9- and 12-months post-surgery.

\section{Patients and Methods}

\section{Study design}

In this cross-sectional analysis, 39 renal transplant candidates were enrolled through Imam-Khomeini hospital complex affiliated with Urmia university of medical sciences. Inclusion criteria were age $>12$ years, resident of West Azerbaijan province. Relevant demographic and clinical data (recipient- and donor age on the day of the surgery, cold ischemia time, type and duration of preoperative dialysis, end-stage renal failure etiology, postoperative daily $\mathrm{sCr}$ and $\mathrm{UO}$ ) were collected from the patients' hospital records. Organ recipients received a triple-drug-based immunosuppressive regimen combining tacrolimus, mycophenolate mofetil and methylprednisolone.

\section{Serum preparation}

Blood ( $4 \mathrm{~mL}$ ) was drawn into a Vacutainer by venipuncture. Sampling was performed at pre-operation day and at 2, $16,24,36$ and 48 hours after surgery. The blood samples were allowed to clot at room temperature for 30 minutes. The samples were then centrifuged at $1500 \mathrm{rpm}$ for 10 minutes. The sera $(0.5 \mathrm{~mL}$ aliquots $)$ were transferred into Eppendorf tubes and subsequently stored at $-70^{\circ} \mathrm{C}$ until further analysis.

\section{Biomarker measurements}

Serum NGAL levels were measured using an ELISA Kit (Shanghai Crystal Day Biotech, China) according to the manufacturer's instructions. Creatinine measurement was performed according to the Jaffe method using a commercial kit (Pars Azmoon Inc., Tehran) on a BT-auto analyzer (13).

\section{Post-operative outcome}

Kidney recipients were categorized into immediate graft function (IGF) and DGF based on estimated glomerular filtration rate (eGFR) using Modification of Diet in Renal Disease (MDRD) equation: GFR $\left(\mathrm{mL} / \mathrm{min}\right.$ per $\left.1.73 \mathrm{~m}^{2}\right)=$ $175 \times$ sCr- $1.154 \times$ age- $0.203 \times 1.212$ (if patient is black) $\times 0.742$ (if female). IGF was defined as an eGFR $>60 \mathrm{~mL} /$ min per $1.73 \mathrm{~m}^{2}$ on day 5 post-surgery.

\section{Statistical analysis}

The data were analyzed using SPSS version 17. Normal distribution continuous variables are presented as mean \pm standard deviation. Non-normal distribution continuous variables are presented as median inter-quartile range variables. Categorical variables were displayed by number and percentage. The Kolmogorov-Smirnov test was used to determine each continuous variable's normal distribution .The student $t$ test or Mann-Whitney $\mathrm{U}$ test measured the difference in continuous variables between two groups and the chi-square test or the Fisher's exact test assessed categorical variables between two groups. To assess the diagnostic significance of the indicators, receiver operating characteristic (ROC) analysis was performed. Optimum cut-off value was determined as described elsewhere (14).

Results

Underlying causes of ESRD were hypertension $(n=22$; 
$56.41 \%)$; diabetes mellitus $(\mathrm{n}=3 ; 7.69 \%)$; glomeruli nephrite $(n=3 ; 7.69 \%)$ and polycystic kidney disease $(n=3$; $7.69 \%)$. In eight kidney recipients (20.51\%), the causes for renal failure were not specified. After transplantation, kidney recipients $(n=14)$ were retrospectively categorized as DGF whilst the reminder organ recipients $(n=25)$ were classified as IGF. Age only was statistically different between DGF- and IGF groups. Characteristics of kidney donors and kidney recipients are given in Table 1.

Pre-surgery, median eGFR (mean; range) in the kidney recipients as a whole was $7.3 \mathrm{~mL} / \mathrm{min}$ per 1.73 $\mathrm{m}^{2}(7.77 \pm 3.30 ; 3.30-16.85)$. In the IGF group, the eGFR progressively increased after surgery, reaching 60 at day 3 post-surgery. A maximum eGFR was obtained at day 14 post-surgery $70.92(62.45 \pm 19.30 ; 23.18$ to 110.13$)$. In the case of the DGF group, an improvement in the eGFR was also improved but at slower rate. At day 14 post-surgery, median eGFR was $46(48.34 \pm 19.31 ; 23.18$ to 81.28$)$.

Trends for eGFR in the IGF- and DGF groups are shown in Figure 1. Comparison of eGFR between IGF and DGF groups revealed that statistical differences were seen at checkpoint 16 hours post-surgery and onwards.

Retrospective classification of the organ recipients according to post-operative outcome showed that median sNGAL (mean; range) in the DGF subset was higher than that of the IGF group $202.35 \mathrm{ng} / \mathrm{mL}(330.80 \pm 341.18$ $\mathrm{ng} / \mathrm{mL})$ versus $124.30 \mathrm{ng} / \mathrm{mL}(294.60 \pm 324.70 \mathrm{ng} / \mathrm{mL})$. Interestingly, a statistical difference was seen at 2 hours post-surgery. Figure 2 displays the trends for sNGAL in the organ recipient during the follow-up.

At 2 hours post-surgery, AUC, sensitivity and specificity for sNGAL were $0.73,100 \%$ and $52 \%$. Figure 3 displays the receiver operating characteristics for SNGAL and eGFR at two hours surgery.

Strong correlations were seen between sNGAL at two hours post-surgery and eGFR at 6,9 and 12 months $(\mathrm{r}=0.66, \quad P=0.007 ; \quad \mathrm{r}=0.836, \quad P=0.031 ; \quad$ and $\mathrm{r}=0.93$; $P=0.016)$.
Table 1. Provides information about the patients' demographic and clinical variables

\begin{tabular}{llll}
\hline Characteristic & DGF (14) & IGF (25) & $P$ value \\
\hline Donor age $(\mathrm{y})$ & $30.43 \pm 10.58$ & $27.68 \pm 4.06$ & 0.71 \\
Recipients age $(\mathrm{y})$ & $47.57 \pm 13.29$ & $38.32 \pm 12.23$ & 0.034 \\
Gender, male $(\mathrm{n})$ & $8(38.09 \%)$ & $13(61.90 \%)$ & 0.064 \\
BMI $\left(\mathrm{kg} / \mathrm{m}^{2}\right)$ & $23.97 \pm 3.7$ & $24.28 \pm 3.97$ & 0.69 \\
Cold ischemic time (min) & $66.64 \pm 20.19$ & $72.84 \pm 18.96$ & 0.27 \\
Time on dialysis (mon) & $15.64 \pm 16.96$ & $19.8 \pm 19.23$ & 0.317 \\
\hline
\end{tabular}

\section{Discussion}

In the current report, frequencies of DGF was $36 \%$ when immediate recovery of renal function was defined as an eGFR $>60 \mathrm{~mL} / \mathrm{min}$ per $1.73 \mathrm{~m}^{2}$ on day 5 post-surgery (15). On the other hand, the frequency of DGF was $17 \%$ when the need for dialysis was defined as an index of postoperative outcome. Our finding is in contrast with the nil percent recently by Buemi et al (12). Of note is that Buemi et al DGF was defined as the requirement for at least one dialysis session during the first week after surgery. In their study, the recipients received antiCD25 monoclonal antibody on days 1 and 4 as induction therapy. The difference in the frequency of postoperative outcome between the aforementioned investigation(s) is possible due to implementation of induction therapy. Taken together, further studies are required to elucidate the cause(s) of the higher rate of DGF our center.

We have also found that preoperative median sNGAL in our cohort by ELISA was 50\% lower than that reported by the Triage NGAL test (12). The latter is a rapid fluorescence immunoassay and is a point-of-care assay. The disparity between our value and that reported by Buemi et al is likely to reflect difference in cold-ischemia time (70 minutes versus 240 minutes).

Thirdly, retrospective segregation of the studied population according to early postoperative outcome

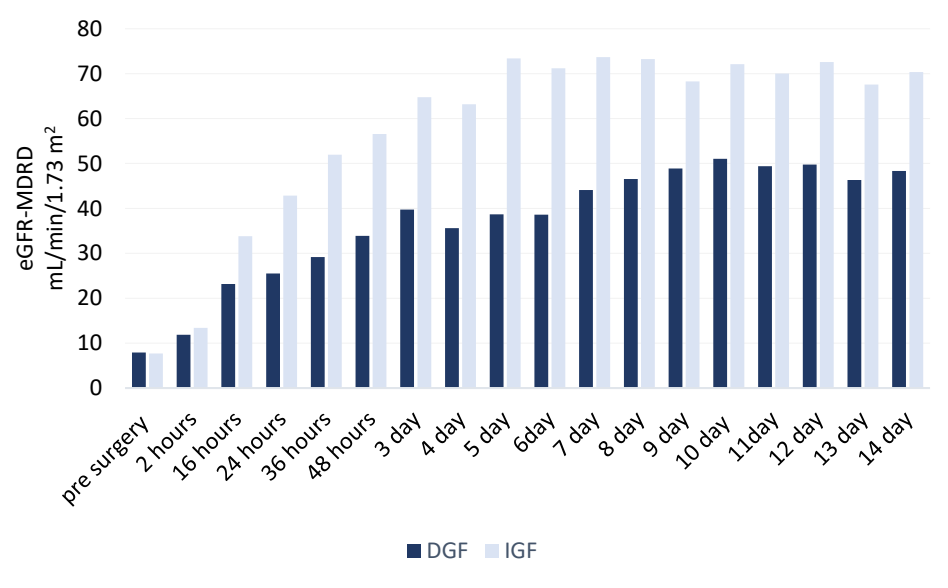

Figure 1. Temporal trends for median estimated glomerular filtration rate - MDRD (modification of diet in renal disease) in the delayed graft function - and immediate graft function groups. 


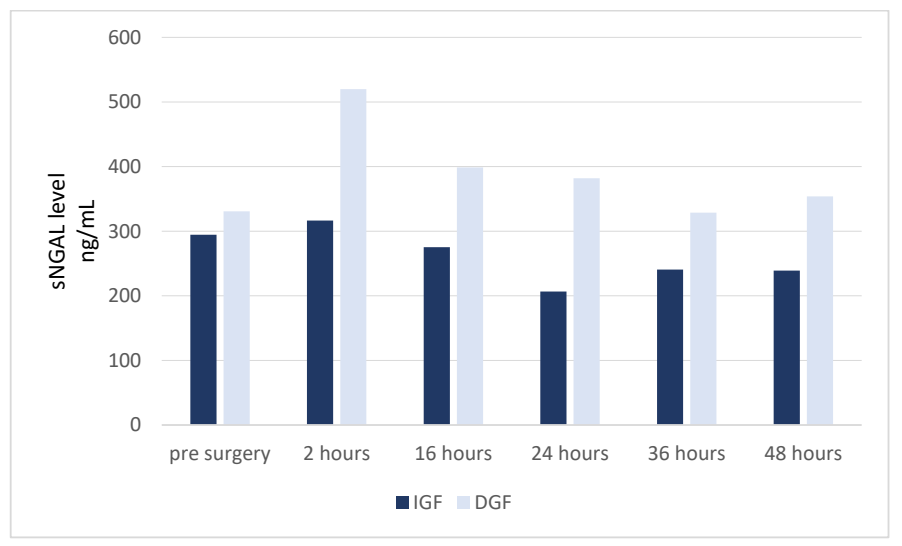

Figure 2. Serum NGAL patterns in the DGF and IGF groups.
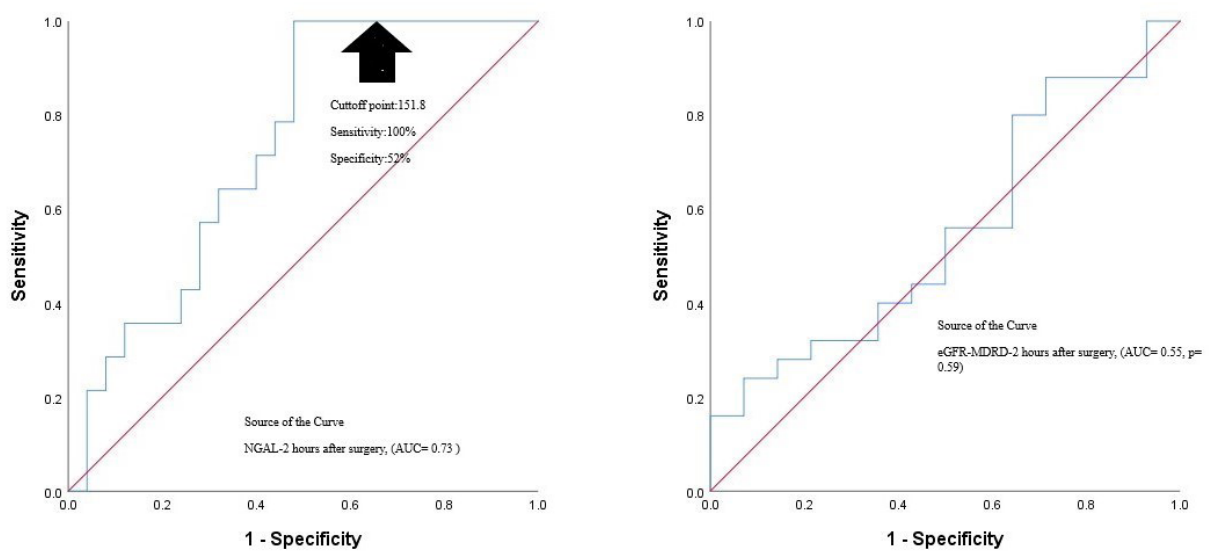

Figure 3. Receiver operating characteristic at 2-hour surgery for sNGAL and eGFR-MDRD.

revealed that median sNGAL in the DGF group, prior to surgery, was $21 \%$ higher than that of the IGF group. Interestingly, this finding is in line with the results of reported by Nielsen et al (11) evaluating diagnostic performance $\mathrm{pNGAL}$ in a cohort of renal transplant patients from brain death donors. A similar phenomenon has been noticed in patients receiving dialysis prior to transplantation (11). Furthermore, higher sNGAL levels has been detected in kidney recipients with underlying risk factors compared to those transplanted preemptive (11). Many organs are capable of expressing NGAL, especially during periods of aggression. Accordingly, NGAL values can be significantly modified by pre-existing conditions in organ donors and organ recipients. Indeed, Portilla et al (16) attributed the paradox to disruption of megalindependent endocytosis in the renal proximal tubule and thus leading to manifestation of higher sNGAL. Taken together these observations imply that donors /recipient dependent factors may be responsible for the elevated sNGAL in the DGF patients. The clinical significance of the elevated sNGAL among the patients with DGF prior to surgery merits further investigation.

\section{Conclusion}

This study is the first to uncover that pre-transplant sNGAL levels in DGF were higher compared to IGF and that sNGAL at 2-hours post-surgery correlates allograft function at 6-, 9- and 12-months. Multicenter study with large samples-size is required to ascertain the usefulness of sNGAL as a diagnostic tool for the prediction of allograft function in renal transplant patients from living donors.

\section{Limitations of the study}

These were; 1) small sample size; 2) a single-center study that needs further validation at a multi-center level or larger sample size is required; and 3) lack of a standardized measure of glomerular filtration rate.

\section{Acknowledgments}

We thank so much for the work and support of all the physicians, nurses and transplant workers of ImamKhomeini hospital complex affiliated with Urmia university of medical sciences.

Authors' contribution

JNZ and ATF were the principal investigators of the study. 
JNZ, EA and FJG were included in preparing the concept and design. All authors participated in preparing the final draft of the manuscript, revised the manuscript and critically evaluated the intellectual contents. All authors have read and approved the content of the manuscript and confirmed the accuracy or integrity of any part of the work.

\section{Conflicts of interest}

The authors declare that they have no competing interests.

\section{Ethical issues}

The research followed the tenets of the Declaration of Helsinki. The institutional ethical committee at Urmia University of Medical Sciences approved all study protocols (Ethical code \#IR.UMSU.REC.1396.62). Accordingly, written informed was consent taken from all participants before any intervention. Additionally, ethical issues (including plagiarism, data fabrication, double publication) have been completely observed by the authors. This study was extracted from MSc thesis of Elham Adlravan at Urmia University of Medical Sciences (Thesis\# 95-01-40-2218).

\section{Funding/Support}

This project was supported by Research and Development Deputy, Urmia University of Medical Sciences (Grant\#183) and National Institute for Medical Research and Development (Grant \# 943780).

\section{References}

1. Wolfe RA, Ashby VB, Milford EL, Ojo AO, Ettenger RE, Agodoa LY, et al. Comparison of mortality in all patients on dialysis, patients on dialysis awaiting transplantation, and recipients of a first cadaveric transplant. N Engl J Med. 1999;341:1725-30. doi: 10.1056/NEJM199912023412303.

2. Yarlagadda SG, Coca SG, Formica RN Jr, Poggio ED, Parikh CRJNdt. Association between delayed graft function and allograft and patient survival: a systematic review and meta-analysis. Nephrol Dial Transplant. 2009;24:1039-47. doi: $10.1093 / \mathrm{ndt} / \mathrm{gfn} 667$.

3. Nashan B, Abbud-Filho M, Citterio FJCt. Prediction, prevention, and management of delayed graft function: where are we now? Clin Transplant. 2016;30:1198-208. doi: $10.1111 /$ ctr. 12832 .

4. Howard R, Pfaff W, Brunson M, Ramos E, Peterson J, editors. Delayed graft function is associated with an increased incidence of occult rejection and results in poorer graft survival. Transplant Proc. 1993;25:884.

5. Mishra J, Ma Q, Kelly C, Mitsnefes M, Mori K, Barasch J, et al. Kidney NGAL is a novel early marker of acute injury following transplantation. Pediatr Nephrol. 2006;21:856-
63. doi: 10.1007/s00467-006-0055-0.

6. Kielar M, Dumnicka P, Gala-Błądzińska A, BędkowskaProkop A, Ignacak E, Maziarz B, et al. Urinary NGAL Measured after the First year post kidney transplantation predicts changes in glomerular filtration over oneyear follow-up. J Clin Med. 2021;10:43. doi: 10.3390/ jcm 10010043 .

7. Klein SJ, Brandtner AK, Lehner GF, Ulmer $H$, Bagshaw SM, Wiedermann CJ, et al. Biomarkers for prediction of renal replacement therapy in acute kidney injury: a systematic review and meta-analysis. Intensive Care Med. 2018;44:323-336. doi: 10.1007/s00134018-5126-8.

8. Malyszko J, Lukaszyk E, Glowinska I, Durlik M. Biomarkers of delayed graft function as a form of acute kidney injury in kidney transplantation. Sci Rep. 2015;5:11684. doi: 10.1038/srep11684.

9. Devarajan PJN. Neutrophil gelatinase-associated lipocalin: A troponin-like biomarker for human acute kidney injury. Nephrology (Carlton). 2010;15:419-28. doi: 10.1111/j.14401797.2010.01317.x.

10. Heyne N, Kemmner S, Schneider C, Nadalin S, Königsrainer A, Häring H-UJT. Urinary neutrophil gelatinase-associated lipocalin accurately detects acute allograft rejection among other causes of acute kidney injury in renal allograft recipients. Transplantation. 2012;93:1252-7. doi:10.1097/ TP.0b013e31824fd892.

11. Nielsen MB, Krogstrup NV, Nieuwenhuijs-Moeke GJ, Oltean M, Dor FJ, Jespersen B, et al. P-NGAL Day 1 predicts early but not one year graft function following deceased donor kidney transplantation-The CONTEXT study. PLoS One. 2019;14:e0212676. doi: 10.1371/journal. pone. 0212676 .

12. Buemi A, Musuamba F, Frederic S, Douhet A, De Meyer $\mathrm{M}$, De Pauw L, et al. Is plasma and urine neutrophil gelatinase-associated lipocalin (NGAL) determination in donors and recipients predictive of renal function after kidney transplantation? Clin Biochem. 2014;47:68-72. doi: 10.1016/j.clinbiochem.2014.06.079.

13. Hoste L, Deiteren K, Pottel H, Callewaert N, Martens FJBn. Routine serum creatinine measurements: how well do we perform? BMC Nephrol. 2015;16:21. doi: 10.1186/s12882015-0012-x.

14. Kamarudin AN, Cox T, Kolamunnage-Dona RJBmrm. Time-dependent ROC curve analysis in medical research: current methods and applications. BMC Med Res Methodol. 2017;17:53. doi: 10.1186/s12874-017-0332-6.

15. Mogulla MR, Bhattacharjya S, Clayton PAJTI. Risk factors for and outcomes of delayed graft function in live donor kidney transplantation-a retrospective study. Transpl Int. 2019;32:1151-60. doi: 10.1111/tri.13472.

16. Portilla D, Dent C, Sugaya T, Nagothu K, Kundi I, Moore $\mathrm{P}$, et al. Liver fatty acid-binding protein as a biomarker of acute kidney injury after cardiac surgery. Kidney Int. 2008;73:465-72. doi: 10.1038/sj.ki.5002721.

Copyright (c) 2021 The Author(s); Published by Nickan Research Institute. This is an open-access article distributed under the terms of the Creative Commons Attribution License (http://creativecommons.org/licenses/by/4.0), which permits unrestricted use, distribution, and reproduction in any medium, provided the original work is properly cited. 